\title{
Possible seismogenic origin of changes in the ULF EM resonant structure observed at Teoloyucan geomagnetic station, Mexico, 1999-2001
}

\author{
A. Kotsarenko ${ }^{1}$, O. Molchanov ${ }^{2}$, R. Pérez Enríquez ${ }^{1}$, J. A. López Cruz-Abeyro ${ }^{1}$, S. Koshevaya ${ }^{3}$, V. Grimalsky ${ }^{4}$, and \\ I. Kremenetsky ${ }^{5}$ \\ ${ }^{1}$ Centro de Geociencias en Juriquilla, UNAM, Apdo Postal 1-742, Centro Queretaro, Querétaro, C.P. 76001, Mexico \\ ${ }^{2}$ Institute of the Physics of the Earth, Moscow, Russia \\ ${ }^{3}$ UAEM, Cuernavaca, Morelos, Mexico \\ ${ }^{4}$ INAOE, Tonantzintla, P.O.51 \& 216, C.P. 72000, Puebla, Pue., Mexico \\ ${ }^{5}$ Institute of Space Research, Nat. Acad. Sci. and Nat. Space Agency, Ukraine
}

Received: 29 June 2005 - Revised: 24 August 2005 - Accepted: 24 August 2005 - Published: 22 September 2005

Part of Special Issue "Seismic hazard evaluation, precursory phenomena and seismo electromagnetics"

\begin{abstract}
The evolution of the ULF resonant structure observed at Teoloyucan geomagnetic station has been provisionally studied in a possible relation with seismic activity in Mexico in the period 1999-2001. Two resonant lines were observed in the H-component (linear polarization) in the frequency bands $f_{R 2}=10.2-11.1 \mathrm{mHz}$ and $f_{R 2}=13.6-14.5$ $\mathrm{mHz}$, sometimes accompanied by other harmonics. The source of the observed resonances is possibly related with the geomagnetic location of the station (geomagnetic latitude $\lambda=29.1^{\circ}$ ) and its proximity to the equatorial electrojet $\left(\lambda \sim 30^{\circ}\right)$. An enhancement of the carrier frequency of both resonances in the period 1 month-2 weeks was found before the strongest EQs. Also, a depression of the resonant structure just a few days before and a few days after some EQs seems to be correlated with seismic activity.
\end{abstract}

\section{Introduction}

Geomagnetic monitoring of tectonically active areas have reported anomaly changes in the character of the geomagnetic field occurring before, during and after some earthquakes (Hayakawa and Molchanov, 2002). In this respect, different theoretical models have been proposed about their origin, to be able to give a physical mechanism to explain the geomagnetic anomalies. Some of these studies emphasize the crustal origin of the source (Surkov et al., 2003), and other argue on their ionospheric pertinence (Pulinets and Boyarchuk, 2004).

The analysis of the seismogenic geomagnetic emission depends strongly on the instrumental infrastructure available.

Correspondence to: A. Kotsarenko

(kotsarenko@geociencias.unam.mx)
In the case of a network of geomagnetic stations, the multistation data set grants the possibility for analysis by means of different robust techniques, such as the location of the area of the geomagnetic disturbances (Kopytenko et al., 2001), and for the separation of the geomagnetic field in its constituents by their statistical properties (for instance Principal Component Analysis (PCA), Hattori et al., 2004). In case of only one station, the data set possibilities for the analysis are lower but some techniques like fractal analysis are still available to evaluate and recognize the anomalous character of the magnetic field that are produced by forthcoming earthquakes. The aim of this study is to present a preliminary analysis of evolution of the ULF resonant structure observed at Teoloyucan geomagnetic station in the period 1999-2001 to find out a possible correlation with the strong earthquakes occurring during that period.

\section{Experiment and methodology}

The analyzed geomagnetic data were recorded at the Teoloyucan station (Central Mexico, geographic coordinates: $99^{\circ} 11^{\prime} 35.735^{\prime \prime} \mathrm{W}, 19^{\circ} 44^{\prime} 45.100^{\prime \prime} \mathrm{N}, 2280 \mathrm{~m}$ height). This station was equipped with a 3-component fluxgate magnetometer designed at UCLA, operating at $1 \mathrm{~Hz}$ sampling rate frequency, with a GPS system for data synchronization.

We analyzed the events with $M_{s}>4$, concentrating on the 7 earthquakes (Table 1) occurring under quiet geomagnetic conditions and with the condition that the Teoloyucan geomagnetic station be inside or close to the EQ preparation zone. The radius of the earthquake preparation zone is estimated by Dobrovolsky formula $\rho=10^{0.43 M_{s}} \mathrm{~km}$, where $M_{S}$ is the magnitude of the earthquake (Pulinets et al., 2004). 
Table 1. The 7 earthquakes during 1999-2001 chosen for analysis. Please note that 2nd and 6th events are slightly over the estimated preparation zone.

\begin{tabular}{rrrrrrrrrrr}
\hline year & month & day & hour & min & latitude & longitude & $M_{S}$ & Depth, km & Distance, km & $\rho, \mathrm{km}$ \\
\hline 1999 & 6 & 15 & 20 & 42 & -97.51 & 18.18 & $\mathbf{7 . 0}$ & 69 & 262 & 1023 \\
1999 & 6 & 21 & 17 & 43 & -101.72 & 17.99 & $\mathbf{5 . 8}$ & 54 & 343 & 312 \\
1999 & 9 & 30 & 16 & 31 & -97.03 & 15.95 & $\mathbf{7 . 5}$ & 16 & 481 & 1678 \\
2000 & 3 & 12 & 3 & 44 & -99.29 & 20.1 & $\mathbf{4 . 1}$ & 5 & 41 & 58 \\
2000 & 8 & 9 & 11 & 41 & -102.66 & 17.99 & $\mathbf{6 . 5}$ & 16 & 428 & 624 \\
2001 & 5 & 19 & 23 & 21 & -105.72 & 18.27 & $\mathbf{6 . 5}$ & 20 & 736 & 624 \\
2001 & 10 & 7 & 21 & 39 & -100.16 & 16.98 & $\mathbf{6 . 1}$ & 10 & 322 & 420 \\
\hline
\end{tabular}

Year/month/day/hour/min are: the exact time of the EQ,

Latitude and longitude: the geographic coordinates of the epicenter,

$M_{S}$ and Depth: magnitude and depth of the EQ,

Distance: the distance between epicenter and Teoloyucan station,

$\rho$ : is the radius of the EQ preparation zone estimated by Dobrovolsky formula.
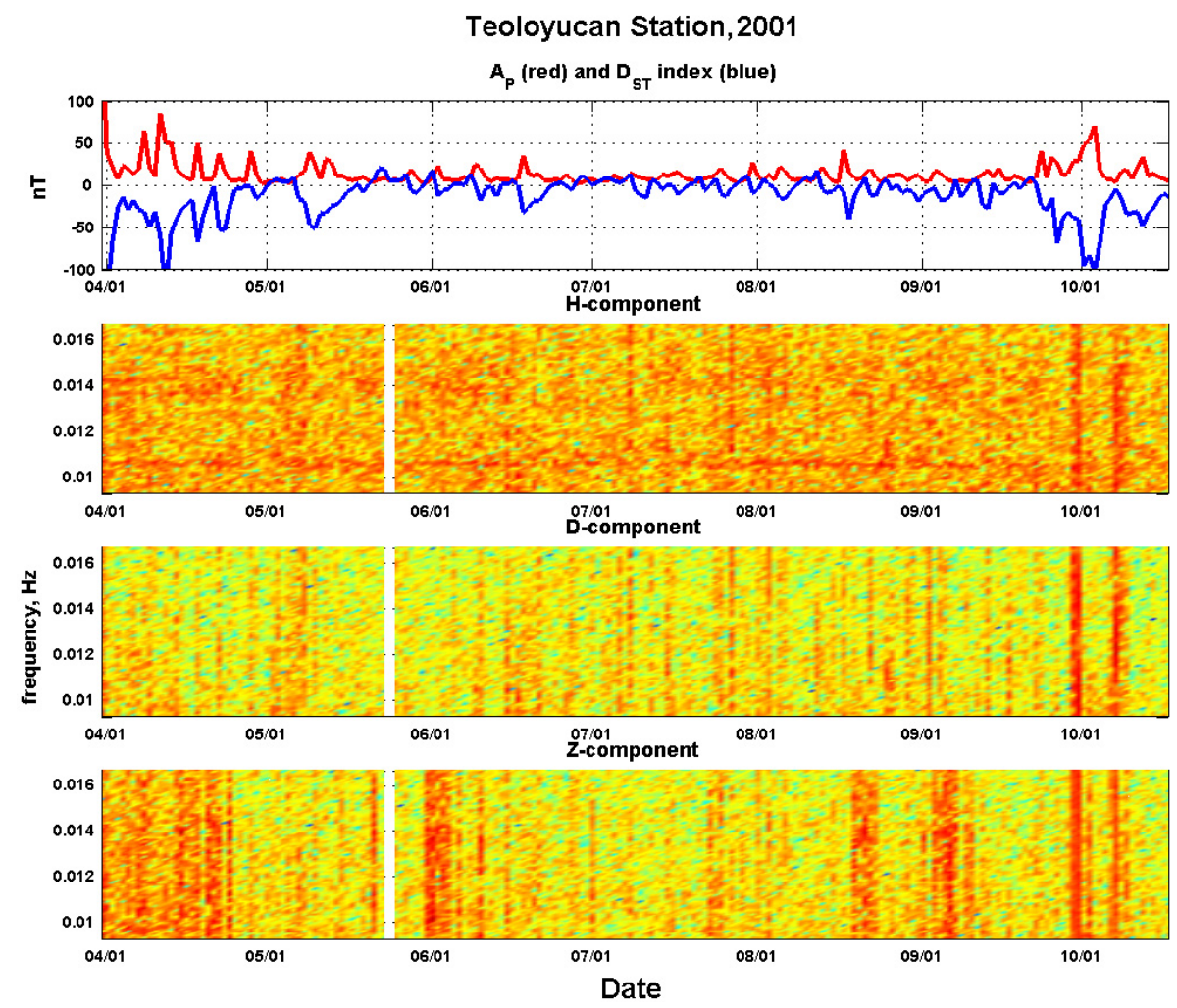

Fig. 1. Observations of the ULF geomagnetic resonances structure in $H$-component, Teoloyucan station, 2001. Time interval 06:0009:00 UT.

Recently, we performed analysis of the continuous part of the geomagnetic spectra by 2 methods: a study of the spectral values $S_{H, D, Z}$ and their ratio $S_{Z} / S_{H}$ as a part of the traditional anlysis, where $H, D$ and $Z$ are the horizontal, declination and vertical components of the magnetic field, and $S$ is calculated as a power spectral density; and a study of the spectral ratio $\beta$ for the fractal analysis (Kotsarenko et al., 2004), where the $\beta$ is calculated as a best linear fit approximation $\log (S)-v s-\log (f)$. In the line spectrum structure, we showed the existence of local geomagnetic pulsa- tions possibly generated by a crustal source. In the present paper we extend our study of the line structure to the analysis of the resonance structure of the geomagnetic field and its possible connection with seismic activity.

The resonance structure of the geomagnetic field has been recently discovered for the data of Teoloyucan magnetic station (paper in preparation). The ULF resonances have been observed in the H-component in the narrow frequency bands $f_{R 2}=10.2-11.1 \mathrm{mHz}$ and $f_{R 2}=13.6-14.5 \mathrm{mHz}$ (Fig. 1). 


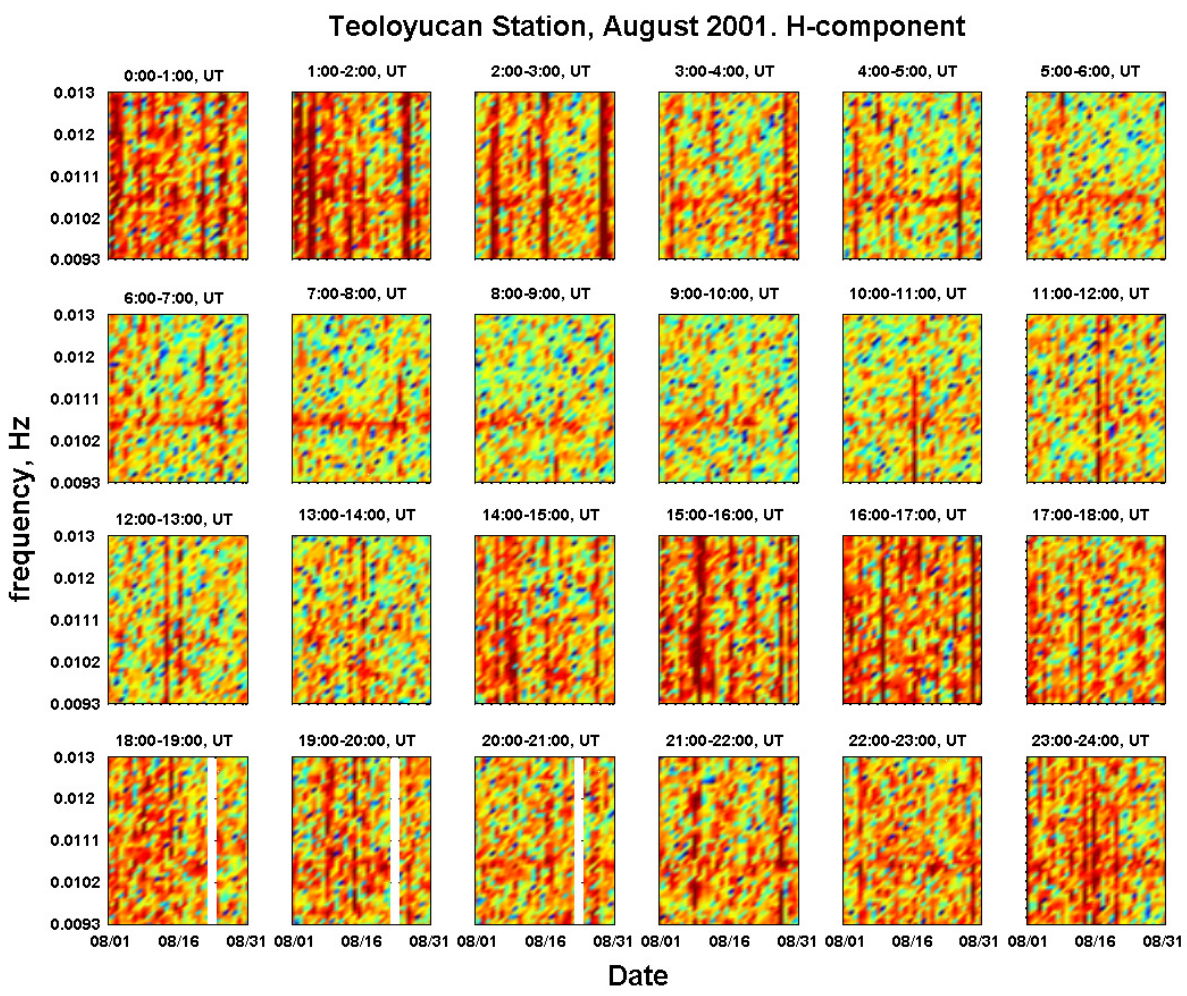

Fig. 2. Appearance of the 1st resonant line in August 2001, in the $H$ geomagnetic component.

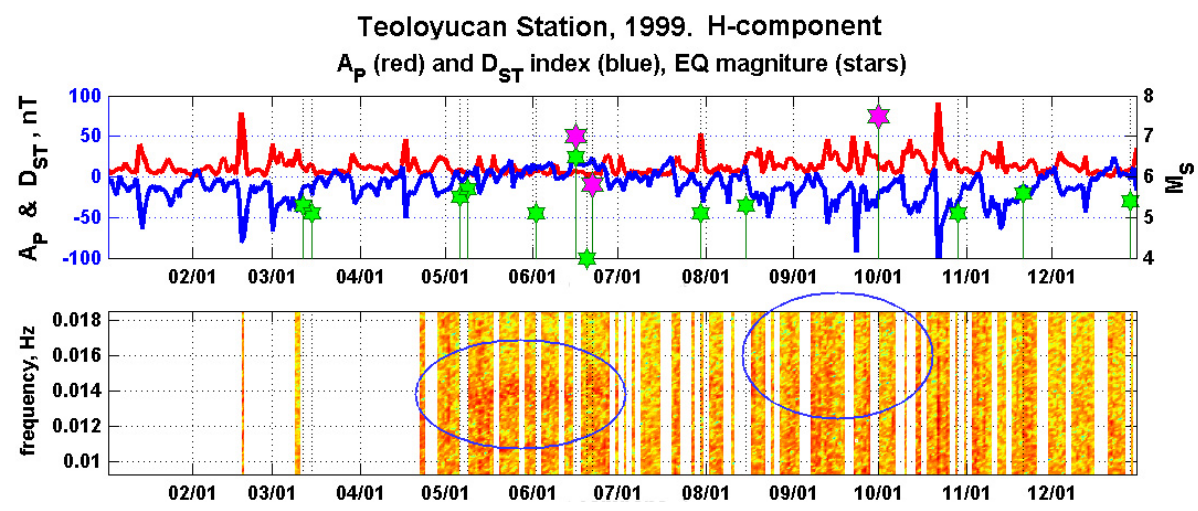

Fig. 3. Year 1999. Upper pannel: $A_{p}$ index (red line), $D_{s t}$ index (blue line) and EQ magnitudes (stars). The EQs with the highest seismic indexes are marked by magenta color. Lower pannel: resonance structure observed in $H$-component. Time interval 06:00-09:00 UT.

These and figures below were obtained in the following way. We divide the day signal into eight 3-hour intervals (1-hour for Fig. 2) and calculate its power spectra with NFFT $=10800$ pts. After that we plot the annual (monthly for Fig. 2) series of spectra related to a particular 3-hour interval as the images in the axes "frequency-vs-time" (days) and color for "intensity", as "regular" spectrograms, obtaining 8 pictures for different local time of the day. Finally, we have chosen the picture related to the time interval 06:0009:00 UT (00:00-03:00 Local Time) for our analysis because it rends the best signal/noise ratio for this period.

The resonance structure has a proper hourly character: it almost disappears during the period 10:00-18:00 UT (Fig. 2), and the central maximum is sometimes followed by higher and lower harmonics (Figs. 1 and 2). The polarization of both resonances is practically linear, and no resonant line is observed either in $Z$ - or $D$-components. In turn, no resonant lines were observed at the nearest to Teoloyucan (TEO) geomagnetic stations, such as Los Alamos (LAL), USA, and Jicamarca (JIC), Peru.

A very similar resonance structure was also observed at Beijing (BJI) geomagnetic station, but it was not found at all even at the nearest to it Chinese station (situated at about $150 \mathrm{~km}$ from Beijing.) The frequency of the first resonant line observed at BJI was the same as that of Teoloyucan, but with an elliptical polarization. 


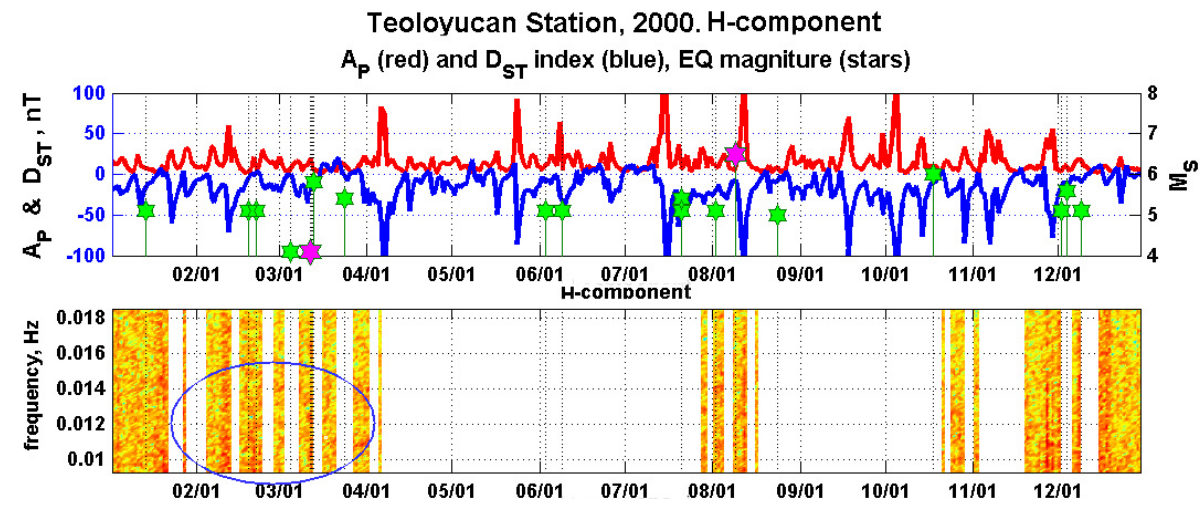

Fig. 4. Year 2000. Upper and Lower pannels: the same as in Fig. 3.

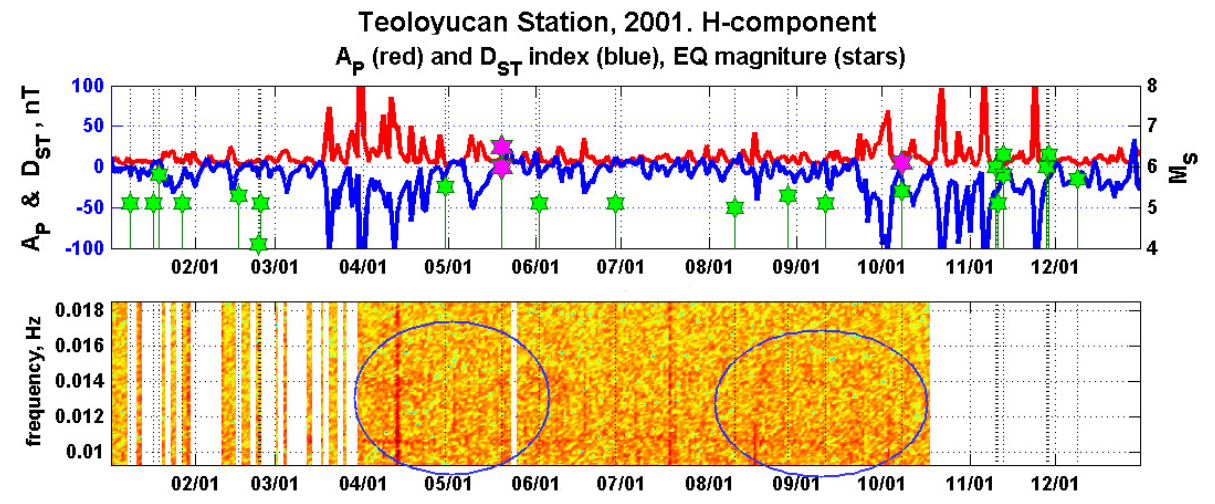

Fig. 5. Year 2001. Upper and Lower pannels: the same as in Fig. 3.

A possible explanation to this phenomenon could be that these stations, TEO and BJI, are located at about the same geomagnetic latitude $\lambda \sim 30^{\circ}$, which coincides with an equatorial electroject displacement. In turn, the absence of a resonant structure at the station closest to Beijing station suggests the presence of a channeling behavior of the observed phenomena. By that, it could be also a longitudinal Alfvén resonance, as the frequency $f=V_{A} / 2 \pi R_{Z}$ for the values of Alfvén velocity $V_{A}=400-600 \mathrm{~km} / \mathrm{s}, f=10-14 \mathrm{mHz}$ fits well with the observed values. Those resonances are expected but almost never observed because of very specific circumstances for their generation, though the transversal Alfvén resonances, or Ionospheric Alfvén Resonances (IAR) are studied quite well (Molchanov et al., 2004).

\section{Results and discussion}

The qualitative analysis of the temporal evolution of the resonant structure show rather interesting tendencies.

Both resonances express a visible increase of carrier frequency until approximately 2 weeks before 2 strongest EQs that occurred in June 1999 (Fig. 3). The same tendency is observed for the EQ that occurred at the end of September, with a higher degree of rise for both resonances.
Unfortunately, due to lack of data for the year 2000 (Fig. 4), we are not able to analyze the strongest EQ of the year that occurred in August. However, we obtain no noticeable increase at the resonant frequencies a week before the series of moderate EQs that occurred in February. Also, we notice that after the last EQ occurring in March both frequencies stabilize.

For the year 2001 we observe an increase of the second resonant frequency until about 1 week before the two strongest EQs occurring in May (Fig. 5). Besides, there was a visible depression of the intensity of both resonant structures just a few days before the EQ occurring at the end of April, and some days after it. A similar depression mechanism but for geomagnetic pulsations was predicted by Sorokin et al. (2003). Another noticeable growth of the second resonant frequency was observed for the period starting about 1 month before the two strongest EQs occurring in October. The first resonance was not stable in this period, but its 2 lower harmonics increase just until the time that the first EQ occurs.

\section{Conclusions}

We studied the evolution of the ULF resonant structure observed at Teoloyucan geomagnetic station in terms of a possible dependence with the strongest earthquakes occurring 
in Mexico in the period of 1999-2001. The ULF resonance structure is observed in the H-component as two resonant packets (linear polarization) in the frequency bands $f_{R 2}=10.2-11.1 \mathrm{mHz}$ and $f_{R 2}=13.6-14.5 \mathrm{mHz}$, with a certain hourly dependence. The probable reason of appearance of the resonant structure can be related to the proximity of the Teoloyucan station to the equatorial electojet $\lambda \sim 30^{\circ}$. The temporal evolution of this structure show a noticeable correlation with EQ activity: a growth of the frequency of both resonances in the period of one month to 2 weeks before the strongest EQs that occurred in June 1999 and about 1 week before the two strongest EQs occurring in May 2001, and a depression of the resonant structure just few days before and few days after some EQs. A more detailed analysis of the observed phenomena will be done in the near future for validation of the obtained results.

Acknowledgements. The authors are grateful to two anonymous referees for suggestions and remarks that have given us the possibility to improve the text of the manuscript.

Edited by: P. F. Biagi

Reviewed by: two referees

\section{References}

Hattori, K., Serita, A, Gotoh, K., et al.: ULF geomagnetic anomaly associated with 2000 Izu islands earthquake swarm, Japan, Phys. Chem. Earth, 29, 425-436, 2004.

Hayakawa, M. and Molchanov, O. A. (Eds.): Seismo-Electromagnetics: Lithosphere-Atmosphere-Ionosphere Coupling, Terra Pub., Tokyo, 353-361, 2002.
Kopytenko, Yu., Ismaguilov, V. S., Kopytenko, Yu. A., Hattori, K., Voronov, P. M., Molchanov, O. A., and Hayakawa, M.: ULF magnetic emissions connected with under sea bottom earthquakes, Nat. Hazards Earth Syst. Sci., 1, 23-31, 2001,

SRef-ID: 1684-9981/nhess/2001-1-23.

Kotsarenko, A., Pérez Enriquez, R., López Cruz-Abeyro, J. A., Koshevaya, S., Grimalsky, V., and Zúñiga, F. R.: Analysis of the ULF electromagnetic emission related to seismic activity, Teoloyucan geomagnetic station, 1998-2001, Nat. Hazards Earth Syst. Sci., 4, 679-684, 2004,

\section{SRef-ID: 1684-9981/nhess/2004-4-679.}

Molchanov, O., Schekotov, A. Yu., Fedorov, E., et al.: Ionospheric Alfvén resonance at middle latitudes: results of observations at Kamchatka, Phys. Chem. Earth, 29, 649-655, 2004.

Pulinets, S. and Boyarchuk, K.: Ionospheric precursors of Earthquakes, Berlin, London, Springer, 2004.

Pulinets, S. A., Liu, J. Y., and Safronova, I. A.. Interpretation of a Statistical Analysis of Variations in the fo F2 Critical Frequency before Earthquakes Based on Data from Chung-Li Ionospheric Station (Taiwan), Geomagnetism and Aeronomy, 44/1, 102-106, 2004.

Sorokin, V., Fedorov, E., Schekotov, A., Molchanov, O., and Hayakawa, M.: The model of seismic related depression of the ULF geomagnetic pulsations, Geophys. Res. Abstr., 5, EAE03A-02190, 2003.

Surkov, V. V., Molchanov, O. A., and Hayakawa, M.: Preearthquake ULF electromagnetic perturbations as a result of inductive seismomagnetic phenomena during microfracturing, J. Atmos. Solar-Terr. Phys., 65, 31-46, 2003. 